Rev. salud pública. 14 (2): 340-349, 2012

\title{
Aspectos jurídicos e sanitários condicionantes para o uso da via judicial no acesso aos medicamentos no Brasil
}

\section{Legal and sanitary aspects conditioning access to medicines in Brazilian courts}

\author{
Mércia Pandolfo $^{1}$, Maria Célia Delduque ${ }^{2}$ e Rita Goreti Amaral ${ }^{1}$ \\ 1 Universidade Federal de Goiás. Goiânia, Brasil.merciap@gmail.com, ritagoreti26@gmail.com \\ 2 Fiocruz. Brasília, Brasil.mcdelduque@gmail.com
}

Recebido 2 Junho 2011/Enviado para Modificação 10 Março 2012/Aprovado 15 Abril 2012

\section{RESUMO}

A busca da garantia do direito à saúde e pelo acesso a medicamentos por intermédio do Poder Judiciário tem sido crescente no Brasil, fenômeno denominado de judicialização da saúde, que tem provocado debates entre operadores do direito, políticos, profissionais e gestores de saúde. Por ser um fenômeno multidimensional, realizou-se uma revisão para discutir os aspectos legais e sanitários que suportam as ações judiciais que demandaram por medicamentos. A saúde é reconhecida como um direito humano fundamental na Constituição Federal Brasileira de 1988 outorgando-Ihe uma proteção jurídica diferenciada no âmbito da ordem jurídicoconstitucional pátria e as garantias ao direito a saúde não estão apenas na Constituição e na lei stricto sensu, mas, principalmente, em um arco normativo infralegal em que se definem as metas e os resultados a serem alcançados pela política pública. As demandas judiciais por medicamentos pode ser um reflexo da dificuldade de acesso aos serviços de saúde, aos vazios e desatualização das ações assistenciais. Para concluir, tal contexto acaba por influir na judicialização da assistência farmacêutica no Brasil.

Palavras-chave: Direito à saúde, assistência farmacêutica, Sistema Único de Saúde, acesso aos serviços de saúde (fonte: DeCS, BIREME).

\section{ABSTRACT}

The search for having access to health care and medicines right granted through Judicial Courts has increased in Brazil. What has been nominated "health judicialization" is a multidimensional phenomenon, a need for dealing with it in a multidisciplinary way involving legal-judicial, political-institutional and sanitary approaches has raised. The Health is recognized as a fundamental human right in the Brazilian Constitution giving it a different legal protection under the legalconstitutional order and the country guarantees the right to health are not only 
the Constitution and the law strictly, but mainly in an normative infralegal arc that define the goals and outcomes to be achieved by public policy. The lawsuits by drugs may be a reflection of the difficulty of access to health services, to empty and downgrading of health care. Therefore, this turns out to affect the judicialization of pharmaceutical care in Brazil.

Key Words: Patient's rights, health policy, health services accessibility/legislation and jurisprudence (source: MeSH, NLM).

\section{RESUMEN}

Condicionantes jurídicos y sanitarios que condicionan el uso de la vía judicial para el acceso a los medicamentos en Brasil

En Brasil ha sido creciente el uso del poder judicial como un medio de garantía del derecho a la salud y el acceso a los medicamentos, fenómeno denominado judicialización de la salud. Esto ha provocado debates entre abogados, políticos, profesionales y gestores de salud. Por tratarse de un fenómeno multidimensional se realizó un revisión para examinar los aspectos legales y sanitarios que soportan las acciones judiciales en las demandas por medicamentos. La salud es reconocida como un derecho humano fundamental en la Constitución Federal Brasilera de 1988 y otorga protección no sólo en el ámbito jurídico constitucional y en las leyes sino también en las políticas públicas, sus metas y productos. Las demandas judiciales por medicamentos pueden reflejar las dificultades en el acceso a los servicios de salud y los vacíos y desactualización de las acciones asistenciales. Para concluir, este contexto influye en la judicialización de la asistencia farmacéutica en Brasil.

Palabras Clave: Derecho a la salud, asistencia farmacéutica, Sistema Único de Salud, acceso a servicios de salud (fuente: DeCS, BIREME).

U m fenômeno que surgiu no início da década de 1990, com os portadores de HIV, e que vem se fortalecendo a partir de então, tem provocado discussões entre juristas, políticos, profissionais e gestores da saúde. Esse fenômeno vem sendo chamado de judicialização da saúde, que consiste na reclamação por bens e serviços de saúde na justiça, por cidadãos brasileiros. Um dos principais produtos de saúde reivindicados nos tribunais são os medicamentos $(1,2)$.

A busca na justiça brasileira pela garantia desse direito não é caso isolado do Brasil. Ações judiciais em países como Peru, Argentina, Venezuela e Equador têm levado os tribunais a deferirem a favor das solicitações, garantindo o acesso a medicamentos e a procedimentos terapêuticos, mesmo quando o direito à saúde não está explícito em suas constituições nacionais $(3,4)$. O que chama a atenção, no caso brasileiro, é o elevado 
grau de sucesso dessas postulações $(5,6)$.

Alguns autores defendem que os mandados judiciais ferem as Diretrizes do Sistema Brasileiro de Saúde (SUS) e a Política Nacional de Medicamentos $(1,7,8)$. Contrariamente, há quem reconheça a legitimidade para o controle das políticas públicas pelo Poder Judiciário, Appio (9), por exemplo, defende que juízes não eleitos podem limitar a vontade de governantes eleitos, e o fazem de forma a ampliar o debate democrático acerca do conteúdo da Constituição.

As discordantes posições na literatura sobre o tema revelam a não consensualidade da questão posta em discussão. Observa-se que o olhar e o entendimento dos operadores do direito, profissionais e gestores de saúde sobre as demandas judiciais divergem e estão relacionados com seus locais de atuação (10), o que não contribui para a racionalização do problema.

A judicialização da saúde envolve aspectos políticos, sociais, éticos, jurídicos e sanitários, por isso sua compreensão envolve, necessariamente, um olhar multidisciplinar. Nesse sentido, este artigo propõe uma revisão narrativa que procurou refletir sobre as demandas judiciais, descrevendo e discutindo os aspectos legais e sanitários, mais especificamente, o acesso aos medicamentos, que dão suporte às ações judiciais que demandaram por medicamentos.

A revisão foi realizada em fontes bibliográficas do tipo primárias, secundárias e em sítios genéricos de busca. Para tanto, utilizaram-se os descritores e termos: "judicialização", "direito à saúde", "demandas judiciais", "constituição federal", "legislação do SUS", "SUS", "políticas de saúde", "política nacional de medicamentos", "assistência farmacêutica", "acesso a medicamentos", "acesso aos serviços de saúde" em português e inglês. As bases de dados consultadas foram LILACS, SciELO, IBECS, MEDLINE.

Direitos à assistência farmacêutica e suas implicações jurisdicionais AConstituição da República Federativa do Brasil (11), em consonância com a evolução constitucional contemporânea e os documentos internacionais, incorporou a saúde e demais direitos sociais não só como bem jurídico e direito social, mas consagrou-a como direito fundamental, outorgandolhe uma proteção jurídica diferenciada no âmbito da ordem jurídico- 
constitucional pátria.

Mas a saúde, além de ser um direito, é também um dever. O artigo 196 da Constituição Federal (11) institui, de um lado, o direito, "A saúde é direito [...]", e o titular desse direito, "[...] de todos [...]", ou seja, todos os brasileiros e estrangeiros residentes no Brasil. Em seguida, nomeia a quem corresponde essa obrigação: “[...] e dever do Estado". A garantia da efetivação desse direito vem com a assertiva "[...] mediante políticas sociais e econômicas que visem a redução do risco de doença e de outros agravos [...]". Assim, se essas políticas não forem desenvolvidas incorrerá o Poder Público em omissão constitucional.

Direito a saúde vai além do conceito de acesso aos estabelecimentos de saúde, inclui uma gama de fatores que podem ajudar a levar uma vida saudável (12). Assim, a norma constitucional impõe ao Estado a realização de políticas públicas que busquem a efetivação deste direito para a população e que mantenham o acesso a ele universal e igualitário.

As demandas judiciais têm sido deferidas sem considerar as políticas públicas de saúde, os princípios e normas do Sistema de Saúde Brasileiro (SUS). Exemplo disso têm sido o deferimento a favor fornecimento de medicamentos que não padronizados nas listas do SUS, como observado em alguns estudos $(1,6,13,14)$.

Assim, a crescente demanda judicial como acesso aos medicamentos representa, de um lado, o exercício efetivo de cidadania por parte da população brasileira e de outro, representa um ponto de tensão perante os elaboradores e executores dessa política no Brasil, que precisam conciliar parcos recursos com a crescente demanda judicial.

Acesso aos medicamentos na realidade brasileira e sua relação com as demandas judiciais

Em todo Brasil pacientes estão procurando os tribunais de justiça para acessar os medicamentos que lhes foram prescritos. Para Bomfim, essas demandas estão assim constituídas: (a) Solicitação de medicamentos disponíveis no SUS - pode refletir dificuldades de acesso; (b) Demandas por medicamentos não disponíveis no SUS-podem estar relacionadas a procedimentos terapêuticos não essenciais ou se referirem a inovações 
tecnológicas não incorporadas pelo SUS; (c) Demandas geradas por condutas médicas discordantes dos protocolos clínicos ou das ações programáticas definidas pelo SUS; (d) Demandas beneficiárias de planos de saúde que solicitam procedimentos que não são cobertos no setor suplementar decorrentes de limitações das coberturas dos planos de saúde.

Solicitação de medicamentos disponíveis no SUS. No Brasil o acesso público aos medicamentos é garantido pela Constituição Federal e pela Lei Orgânica da Saúde e estão disponíveis por componentes: da atenção primária a saúde, de medicamentos estratégicos e especializado da assistência farmacêutica.

No entanto, estudos demonstram que inúmeras ações judiciais têm solicitado medicamentos que fazem partem das listas oficiais de distribuição pública no SUS $(1,2,6,13,15,16)$ indicando deficiência no acesso da população a esses medicamentos.

O acesso aos serviços de saúde no Brasil ainda é crítico por várias razões, inclusive econômicas ou geográficas (17). O padrão de acesso aos serviços públicos de saúde é fortemente influenciado pela condição social das pessoas e pela disponibilidade de acesso à saúde complementar $(18,19)$. A demora no atendimento, o excesso de encaminhamento a outros serviços, a falta de médicos e as deficiências na estrutura física são aspectos apontados pelos usuários como desfavoráveis à utilização do sistema de saúde público (20).

No caso dos medicamentos um estudo realizado em diferentes regiões do Brasil demonstrou que a porcentagem de medicamentos efetivamente dispensados aos usuários varia de 55 a $81 \%$ dos medicamentos prescritos nas unidades de atenção primária (21) indicando uma cobertura ineficiente dos principais problemas de saúde da população.

Mesmo os tratamentos contemplados em programas de atenção à saúde pública podem enfrentar dificuldades de acesso devido à falta de sistemas estáveis de suprimento de medicamentos $(22,23)$. A disponibilidade média percentual em unidades de saúde pública brasileira de um conjunto dos principais medicamentos varia de $46,9 \%$ a $75 \%$ (23) e o tempo médio de desabastecimento nas unidades de saúde é de 84 dias (22). 
A solicitação por via judicial de medicamentos que compõem as listas oficiais de distribuição pública pode representar não apenas falha na disponibilidade desses medicamentos nas unidades de saúde, mas a dificuldade de acesso da população a esses serviços em seu sentido mais amplo, levando em conta o grau de ajuste entre clientes e sistema de saúde (24).

Demandas por medicamentos não disponíveis no SUS. Em proporções variadas, a solicitação de medicamentos que não constam nas listas públicas de medicamentos foi constatada em diversos estudos $(1,2,6,13,15,16)$. Pepe e colaboradores (25) verificaram que $80 \%$ das ações contra o estado do Rio de Janeiro continha solicitação de medicamentos não padronizados.

A solicitação de medicamentos não padronizados pode refletir vazios assistenciais ou a demora do sistema público de saúde na incorporação de novas tecnologias. O SUS está organizado em níveis assistenciais, nos quais cada nível de atenção corresponde a um grupo de procedimentos e serviços adequados aos problemas de saúde da população, estratificados por grau de complexidade e especialidade e os medicamentos são distribuídos através dos diversos programas e ações de recuperação da saúde $(26,27)$. A não oferta de bens e serviços, em qualquer um dos níveis de atenção, configura vazios assistenciais.

A manutenção da oferta de bens e serviços nos diversos níveis de atenção requer o emprego recursos financeiros. Os gastos com medicamentos é um importante elemento de despesas (26), por isso o Poder Executivo vê a obrigação de racionalizá-los a fim de promover ações estruturantes da assistência farmacêutica, fundamentadas nos princípios da universalidade, da integralidade e da equidade, fixados na Lei Orgânica da Saúde (27), para a execução das ações e serviços de saúde. Por isso, entre outras razões, o elenco de medicamentos distribuído gratuitamente se baseia no conceito de medicamentos essenciais.

Para a OMS, "Medicamentos essenciais são aqueles que satisfazem às necessidades prioritárias de cuidados da saúde da população" (28), mas na visão do Poder Judiciário brasileiro medicamento essencial é aquele prescrito pelo médico como necessário a manutenção da saúde do paciente (5).

Essa visão abre espaço para que a indústria farmacêutica utilize-se da via judicial como uma forma de inserção de seus produtos no mercado 
(2), particularmente os mais caros, considerando que o prescritor médico é sensível ao marketing farmacêutico (29-32).

Demandas geradas por conduta médica discordantes dos protocolos clínicos ou das ações programáticas definidas pelo SUS. Estudos sobre demandas judiciais demonstraram que, entre os medicamentos solicitados, boa parcela deles encontrava-se disponível no SUS, inclusive na lista do Componente Especializado de Assistência Farmacêutica $(1,2,6,13,15,16)$.

Esse componente é representado, principalmente, por um grupo de medicamentos destinados ao tratamento de patologias específicas, que atingem um número limitado de usuários e cuja dispensação está condicionada ao atendimento de protocolos clínicos e diretrizes terapêuticas, conforme regulação (32).

Protocolos clínicos são consensos na condução da terapêutica para determinada patologia e são elaborados a partir de evidências científicas e promovem o uso racional de medicamentos. No entanto, esses protocolos e diretrizes são úteis quando constantemente atualizados. Vieira e Zucchi (33), ao analisarem a cobertura das políticas de saúde, concluíram que os protocolos estabelecidos para algumas doenças são deficientes, podendo comprometer a qualidade da assistência terapêutica e indicando necessidade de atualização.

Demanda beneficiária de planos de saúde que solicitam procedimentos que não são cobertos no setor suplementar decorrentes de limitações das coberturas dos planos de saúde. Analisando os medicamentos solicitados em ações judiciais descritos em vários estudos, foram identificados medicamentos que estão ligados a procedimentos hospitalares, como tratamentos oncológicos, antibioticoterapia intravenosa, dietas parenterais $(1,2,6,7,22)$.

A CF88 prevê a participação da iniciativa privada de forma suplementar ao SUS. Assim, uma parcela da população utiliza os serviços privados com desembolso próprio ou por meio de seguros de saúde. Essa opção da população é incentivada pelo Estado brasileiro através de renúncias fiscais (34).

Porém, não é incomum os planos de saúde negarem o pagamento de determinados procedimentos. Alves, Bahia e Barrosos (35) estudaram 
decisões judiciais relacionadas aos planos e seguros privados de saúde e descobriram que a falta de cobertura é uma das principais reclamações dos usuários desse tipo de serviço.

ACF88 representa uma conquista da sociedade brasileira, um avanço no processo político-social. A Constituição e a Lei 8080 puseram em prática o ousado projeto de reforma sanitária, reconhecendo a saúde como direito de cidadania. No entanto, a falta de legislação infraconstitucional deixa lacunas para o adequado entendimento dos conceitos de universalidade e integralidade, que refletem na fragilidade do sistema de financiamento da saúde.

Para melhoria e ampliação das ações e serviços de saúde é essencial que o SUS receba financiamento compatível com a superação dos vazios assistenciais e das desigualdades regionais e setoriais que ainda caracterizam o acesso e a utilização de serviços. Mas há de se ter claro que, mesmo com mais aporte financeiro, sempre se terá de viver com um limite orçamentário que obrigará as escolhas. É dever do Poder Executivo fazêlas de modo a promover a equidade e o bem-estar coletivo.

Assim, é nítida a necessidade da aproximação do direito e da saúde coletiva, em uma agenda única de saúde que discuta e proponha ações que permitam que sejam alcançados resultados capazes de assegurar a tutela dos direitos à saúde de modo eficiente, sem a excessiva interferência do Poder Judiciário nas políticas públicas de saúde e na gestão do SUS

\section{REFERÊNCIAS}

1. Vieira FS, Zucchi P. Distorções causadas pelas ações judiciais à política de medicamentos no Brasil. Rev Saúde Pública 2007; 41(2):214-22.

2. Chieffi $A L$, Barata RB. Judicialização da política pública de assistência farmacêutica e equidade. Cad Saúde Pública 2009; 25(8):1839-1849.

3. Byrne I. Enforcing the right to health: innovative lessons from domestics courts. In: Soto, $\mathrm{H}$; Cheneval, F. Swiss: Swiss Human Right Book [Internet]. Disponible en: http://www. Swisshumanrightsbook.com/SHRB/shrb_03_files/37_453_ Byrne.pdf. Consultado en: Julio 2009.

4. Hogerzeil HV, Samson M, Casanovas JV, Rahmani-Ocara L. Is access to essential medicines as part of the fulfillment of the right to health enforceable through the courts? The Lancet. Jul 22, 368:305-311. [Internet]. Disponible en: www.thelancet. com. Consultado em julio 2009.

5. Marques SB, Dallari SG. Garantia do direito social à assistência farmacêutica no Estado de São Paulo. Rev Saúde Pública 2007; 41(1):1017. 
6. Borges DCL, Ugá MAD. Conflitos e impasses da judicialização na obtenção de medicamentos: as decisões de $1^{\mathrm{a}}$ instância nas ações individuais contra o Estado de Rio de Janeiro, Brasil, em 2005. Cad. Saúde Pública 2010; 26(1):59-69.

7. Afonso Silva V, Terrazas FV. Claiming the Right to Health in Brazilian Courts: The Exclusion of the Already Excluded. SSRN 2009. [Internet]. Disponible en: http://ssrn. com/abstract=1133620. Consultado en Julio 2009.

8. Campilongo CF. Política, sistema jurídico e decisão judicial. São Paulo: Max Limonad; 2002.

9. Appio E. Controle Judicial das Políticas Públicas no Brasil. Porto Alegre: Juruá; 2007.

10. Freire AMP. Constitucionalismo garantista y democracia. Revista Crítica Jurídica [Internet]. Disponible en: http://www.unibrasil.com.br/ detalhe_categoria.asp?id=446. Consultado en: diciembre 2007.

11. BRASIL. Constituição (1988). Constituição da República Federativa do Brasil. Brasília, DF: Senado; 1988.

12. Maués AGM, Simões SAS. Direito público sanitário constitucional. En: Curso de especialização a distância em direito sanitário para membros do Ministério Público e da Magistratura Federal. Brasília: Ministério da Saúde 2002.

13. Messeder AM, Osorio-de-Castro CGS, Luiza VL. Mandados judiciais como ferramenta para garantia do acesso a medicamentos no setor público: a experiência do estado do Rio de Janeiro, Brasil. Cad Saúde Pública 2005; 21(2):525-534.

14. Vieira FS. Ações judiciais e direito à saúde: reflexões sobre a observância aos princípios do SUS. Rev Saúde Pública 2008; 42(2):365-369.

15. Pereira JR, Santos RI, Nascimento Junior JM, Schenkel EP. Análise das demandas judiciais para o fornecimento de medicamentos pela Secretaria de Estado da Saúde de Santa Catarina nos anos de 2003 e 2004. Rev C S Col; 2007. [Internet]. Disponible en: http://www.abrasco.org.br/. Consultado en: marzo 2008.

16. Leite SN, Pereira SMP, Silva P, Nascimento-Jr JM, Cordeiro BC, Veber AP. Ações judiciais e demandas administrativas na garantia do direito de acesso a medicamentos em Florianópolis-SC. Revista de Direito Sanitário 2009; 10(2):13-28.

17. Comissão Nacional sobre determinantes sociais. As causas sociais das iniqüidades em saúde no Brasil: relatório final da Comissão Nacional Sobre Determinantes Sociais da Saúde (CNDSS); 2008. [Internet]. Disponible en: www.cndss.fiocruz.br/pdf/home/ relatorio.pdf. Consultado en: febrero 2008.

18. Travassos C. Equidade e o Sistema Único de Saúde: uma contribuição para debate. Cad. Saúde Pública; 1997. [Internet]. Disponible en: http://www.scielo.br/scielo. php?script=sci_arttext\&pid=S0102-311X1997000200024\&lng=pt\&nrm=iso\&tlng=pt. Consultado en: octubre 2010.

19. Silva NN, Pedroso GC, Puccini RF, Furlani WJ. Desigualdades sociais e uso de services de saúde: evidências de análise estratificada. Rev Saúde Pública 2000; 34(1):44-49.

20. Schwartz TD, Ferreira JTB, Maciel ELN, Lima RCDL. Estratégia saúde da família: avaliando o acesso ao SUS a partir da percepção dos usuários da Unidade de Saúde de Resistência, na região de São Pedro, no município de Vitória (ES). Ciência \& Saúde Coletiva 2010; 15(4):2145-2154.

21. Karnikowski MGO, Nóbrega OT, Naves JOS, Silver LD. Access to Essential Drugs in 11 Brazilian Cities: a community-based evaluation and action method. J Public Health Policy 2004; 25(3-4):288-298.

22. Organização Pan-Americana da Saúde. Avaliação da Assistência Farmacêutica no Brasil / Organização Pan-Americana da Saúde, Organização Mundial da Saúde; Ministério da Saúde - Brasília: Organização Pan-Americana da Saúde; Brasil: Ministério da Saúde, 2005.

23. Naves JOS, Silver LD. Avaliação da assistência farmacêutica na atenção primária no Distrito Federal. Rev Saúde Pública; 2005. [Internet]. Disponible: http://www. scielo.br/scielo.php?pid=S0034-89102005000200013\&script=sci_abstract\&tlng=pt. 
Consultado en: diciembre 2009

24. Penchansky DBA, Thomas JW. The concept of access: definition and relationship to consumer satisfaction. Med Care 1981;19:127.

25. Pepe VLE, Ventura M, Santana JMB, Figueiredo TA, Souza VR, Simas L, et al. Cad Saúde Pública 2010; 26(3):461-471.

26. Brasil. Conselho Nacional de Secretários de Saúde. Assistência farmacêutica no SUS. Brasília: CONASS; 2007.

27. Brasil. Lei 8.080 de 19 de setembro de 1990. Dispõe sobre as condições para a promoção, proteção e recuperação da saúde, a organização e o funcionamento dos serviços correspondentes e dá outras providências. [Internet]. Disponible en http:// www.planalto.gov.br/ccivil_03/leis/l8080.htm. Consultado en: febrero 2010.

28. World Health Organization. Report on the 12th Expert Committee on the Selection and Use of Essential Medicines: technical report series; 2003. [Internet]. Disponible en: http://apps.who.int/medicinedocs/en/d/Js4875e/5.html. Consultado en: septiembre 2009.

29. Theodouro M, Tsiantou V, Pavlakis A, Maniadakis N, Fragoulakis V, Pavi E, Kyriopoulos J. Factors influencing prescribing behaviour of physicians in Greece and Cyprus: results from a questionnaire based survey. BMC Health Serv Res; 9(1):150; 2009. [Internet]. Disponible en: http://www.biomedcentral.com/1472-6963/9/150. Consultado en: septiembre 2009.

30. Dorn SD, Farley JF, Hansen RA, Shah ND, Sandler RS. Direct-to-consumer and physician promotion of tegaserod correlated with physician visits, diagnoses, and prescriptions. Gastroenterology 2009; 137(2):518-24.

31. Fuchs FD. The corporative bias and the molding of prescription practices: the case of hypertension. Braz J Med Biol Res 2009; 42(3):224-228.

32. Barros JAC, Joany S. Anúncios de medicamentos em revistas médicas: ajudando a promover a boa prescrição? Cien Saude Colet 2002; 7(4):891-898.

33. Vieira FS, Zucchi P. Patient lawsuits and treatment provision on the Brazilian National Health Service. Rev Assoc Med Bras 2010; 56(6):672-83.

34. Carvalho EB, Cecílio LCO. A regulamentação do setor de saúde suplementar no Brasil: a reconstrução de uma história de disputas. Cad Saúde Pública 2007; 23:2167-77.

35. Alves DC, Bahia L, Barrosos AF. O papel da justiça nos planos de saúde no Brasil. CAD. Saúde Pública 2009; 25(2):279-290. 